\title{
Design Methodology for Common-Mode Stability of OTA-based Gyrators
}

\author{
Saeed Ghamari, Gabriele Tasselli, Cyril Botteron, and Pierre-André Farine
}

\begin{abstract}
This paper presents a design methodology for common-mode (CM) stability of operational transconductance amplifier (OTA)-based gyrators. The topology of $g_{m}-C$ active inductors is briefly reviewed. Subsequently, a comprehensive mathematical analysis on the CM stability of OTA-based gyrators is presented. Sufficient requirements for the gyrator's CM stability, that easily can be considered during the design process of common-mode feedback (CMFB) amplifiers, are defined. Based on these stability requirements, a design methodology and a design procedure are proposed. Finally, in order to validate the proposed procedure, a resonator with $20 \mathrm{MHz}$ resonance frequency and a quality factor of 20 is fabricated with UMC 180-nm CMOS technology and its CM stability is examined.
\end{abstract}

Keywords-Common-mode stability, $g_{m}-C$ filters, gyrator, operational transconductance amplifier (OTA), resonator.

\section{INTRODUCTION}

Continuous-time filters are widely employed in system-onchip wireless communication systems, such as LTE cellular, GNSS and UWB [1]-[4]. These systems require more and more bandwidth (BW) and selectivity due to the growing number of users and systems working together. These requirements thus also apply to the constituent continuous-time filters. At base-band or relatively low frequencies, active inductors are commonly used in continuous-time filers because they occupy less chip area and provide variable and large inductances as well as tunable quality factors [5]. Active inductors can be realized by transforming the impedance of a capacitor by means of an active device.

Active gyrators provide the most direct way of realizing an inductor and are commonly used in active filter designs [6][8]. They usually consist of two identical operational transconductance amplifiers (OTAs) which are connected together in a negative feedback loop as shown in Figure 1. In theory, by loading an ideal gyrator $\left(c_{p}=0\right.$ and $r_{o} \rightarrow \infty$ in Figure 1) with a capacitor $c_{L}$, the active inductance value will be equal to $L=c_{L} / g_{m}{ }^{2}$, where $g_{m}$ is the transconductance of the OTAs.

Furthermore, system-on-chip integration demands that a fully differential configuration be able to reject the CM disturbances generated by the digital circuitries, clock drivers, substrate coupling and so on. On the other hand, CMFB amplifiers are an inevitable part of any fully differential amplifiers.

S. Ghamari, G. Tasselli, C. Botteron and P.-A. Farine are with the Electronics and Signal Processing Laboratory, École polytechnique fédérale de Lausanne, 2000 Neuchâtel, Switzerland (e-mail: saeed.ghamari@epfl.ch).

Manuscript revised August 11, 2015; accepted August 25, 2015. Early view access on September 22, 2015.

Published online in Wiley Online Library (wileyonlinelibrary.com). DOI: $10.1002 /$ cta. 2154

Copyright (c)2014 John Wiley \& Sons, Ltd.

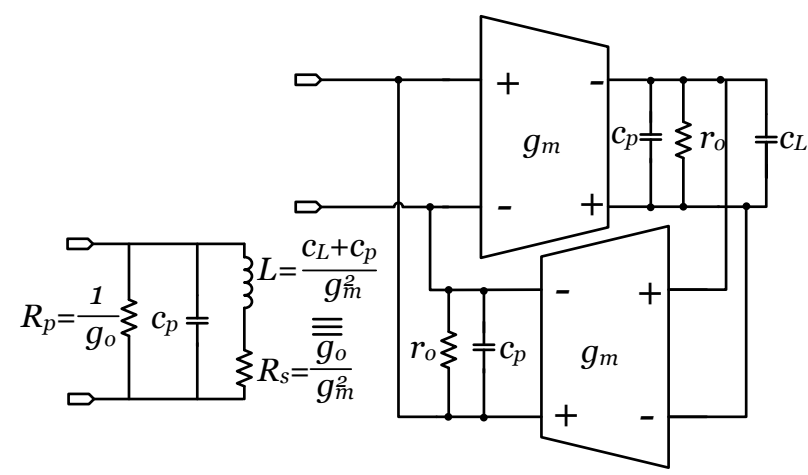

Figure 1. Gyrator-based active inductor with its equivalent RLC circuit; $c_{p}$ and $r_{o}=1 / g_{o}$ are output parasitic capacitance and output resistance of the OTA, respectively.

Therefore, the CM stability of OTAs and gyrators becomes an issue for the design. Although the two OTAs in Figure 1 are in negative feedback for their differential signals, the loop still acts as a positive feedback for CM signals. This positive feedback may cause CM oscillation or latching in a gyrator. $\mathrm{CM}$ stability is the main problem in gyrator design and is not well documented in the literature [9], [10].

This paper proposes, for the first time, a comprehensive analysis that accounts for the CM stability of the OTA-based gyrators. A CM equivalent model of an OTA and a gyrator are used for the analysis. It provides CM stability requirements which easily can be considered during the design process of a CMFB amplifier. Moreover, based on these requirements and considering all the practical issues, a design methodology for CM stability of OTA-based gyrators is also presented. Finally, the developed methodology is validated by simulations and measurements. For this purpose, a resonator with $20 \mathrm{MHz}$ resonance frequency and a quality factor of 20 is fabricated using the UMC 180-nm CMOS technology and fully characterized [11].

The remainder of this paper is organized as follows. Mathematical model and derivation for the CM stability analysis of a gyrator is presented in section II. A design methodology to ensure the CM stability of a resonator is proposed in section III. Section IV presents a practical design procedure based on the proposed methodology. A case study is demonstrated in section V. Finally, section VI provides the conclusions.

\section{Common-Mode Stability: Modeling And ANALYSIS}

Fully differential OTAs are required to be able to reject the $\mathrm{CM}$ disturbances of the circuit and they need CMFB amplifiers 
to regulate their output $\mathrm{CM}$ voltage. The input and output of the CMFB amplifier are the output of the main amplifier. This means that the CMFB amplifier is connected in unity feedback. As a result, its stabilization is usually troublesome since amplifiers are more prone to instability when they are configured as a unity feedback, thus it might require more power to ensure the stability. Besides that, there is a feedback loop in the gyrator (Figure 1) which consists of two OTAs. Without a proper CMFB amplifier for the OTAs, this loop can act as a positive feedback from the CM point of view which may cause a CM oscillation or latching in the gyrator. This oscillation happens inside the loop of the gyrator and is different from the CM oscillation that can happen inside an OTA due to an improper CMFB amplifier. Figure 2 shows the equivalent $\mathrm{CM}$ representation of a gyrator consisting of two folded cascode OTAs with their CMFB amplifiers. The path of the CM positive feedback of the gyrator is shown with a dashed line. This positive feedback is the reason for more stringent requirements for the OTA and its CMFB amplifier in a gyrator loop. In the remainder of this section, we analyze the CM stability of a gyrator by means of a mathematical model and provide stability conditions for it.

\section{A. Common-Mode Stability of an OTA}

The block diagram of an OTA and its CMFB amplifier is shown in Figure 3. Since the focus of this section is on the CM, $G_{C M}(j \omega)$ in Figure 3 is the CM transfer function of a differential OTA without a CMFB amplifier, and $F(j \omega)$ is its CMFB amplifier. For simplicity, it is assumed that the $G_{C M}(j \omega)$ has two poles, one dominant due to the capacitive loading of the OTA $\left(\omega_{1}\right)$ and an internal pole $\left(\omega_{2}\right)$. The same assumption can be used for $F(j \omega)$; apart from an internal pole $\left(\omega_{4}\right), F(j \omega)$ has a dominant pole $\left(\omega_{3}\right)$ which is due to its capacitive loading. Furthermore, $G_{0}$ and $k$ are the DC gain of $G_{C M}(j \omega)$ and $F(j \omega)$, respectively. Therefore, the overall CM transfer function of the OTA in Figure 3 can be written as:

$$
H(j \omega)=\frac{G_{C M}(j \omega)}{1+F(j \omega)},
$$

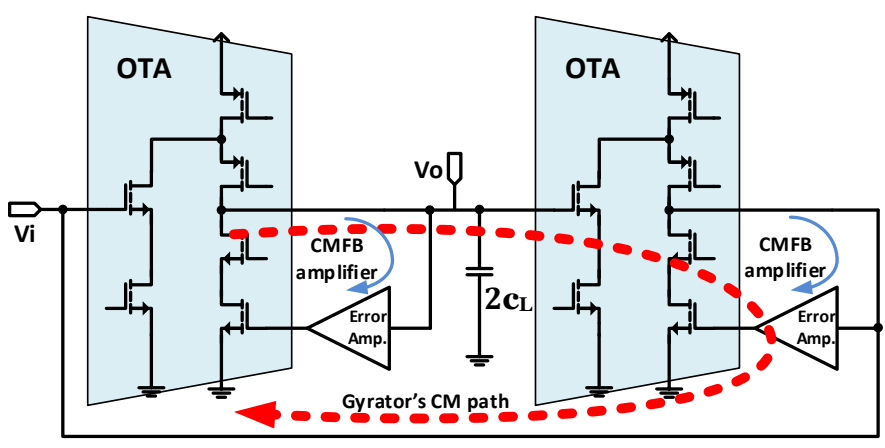

Figure 2. CM equivalent circuit of a gyrator realized by two OTAs; gyrator's CM path is shown by dashed line.

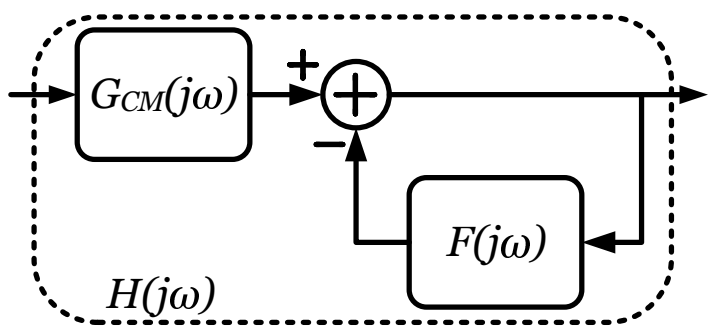

Figure 3. Block diagram of a CM representation of an OTA with its CMFB amplifier $F(j \omega) ; G_{C M}(j \omega)$ is the CM transfer function of the OTA without its CMFB amplifier.

where

$$
\begin{aligned}
& G_{C M}(j \omega)=\frac{G_{0}}{\left(1+j \omega / \omega_{1}\right)\left(1+j \omega / \omega_{2}\right)} \quad \omega_{2}>\omega_{1}, \\
& F(j \omega)=\frac{k}{\left(1+j \omega / \omega_{3}\right)\left(1+j \omega / \omega_{4}\right)} \quad \omega_{4}>\omega_{3} .
\end{aligned}
$$

Assuming that the OTA has been designed for differential mode performance and it is stable, then the values of $\omega_{1}, \omega_{2}$ and $G_{0}$ are fixed, which leaves us with $\omega_{3}, \omega_{4}$ and $k$ to be set for the design of a CMFB amplifier. Note that $G_{C M}(j \omega)$ and $F(j \omega)$ see the same load because they share the loading stage and their outputs are at the same point (assuming the loading capacitors are connected to the ground to provide the path for the CM signal). Accordingly, the dominant pole of $G_{C M}(j \omega)$ and $F(j \omega)$ are the same $\left(\omega_{1}=\omega_{3}\right)$ which leaves us with only $\omega_{4}$ and $k$ to be defined. Considering (1), $H(j \omega)$ is a negative feedback system and one way to examine its stability is to draw the Bode plot of its loop gain $(F(j \omega))$ which for the two poles system of (3) is straightforward. The parameter $k$ is the DC gain of the CMFB amplifier which sets the error of the CM output voltage of the OTA. It also sets the commonmode gain-bandwidth product $\left(\mathrm{GBW}_{\mathrm{CM}}\right)$ of the OTA since $\omega_{3}$ is already set. The higher the $k$ is, the lower the error of the $\mathrm{CM}$ voltage and the higher the $\mathrm{GBW}_{\mathrm{CM}}$ will be. Although a larger value of $k$ is desired, increasing $k$ increases the power consumption of the CMFB amplifier. Therefore, the minimum value for $k$ can be chosen based on the required $\mathrm{GBW}_{\mathrm{CM}}$. For a chosen value of $k$ the position of $\omega_{4}$ sets the phase margin (PM) of the CMFB amplifier, and thus plays the main role in the CM stability of the OTA. For the sake of stability, $\omega_{4}$ should be placed beyond the $\mathrm{GBW}_{\mathrm{CM}}$, thus requiring more power. However, the common technique to reduce this power is to set $\omega_{4}$ lower than $\mathrm{GBW}_{\mathrm{CM}}$ and introduce a zero at $\omega_{5}$ (i.e. by means of a compensation capacitor) into the transfer function of $F(j \omega)$ to gain enough PM to ensure the stability of $H(j \omega)$. In this case, $F(j \omega)$ can be rewritten as:

$$
F(j \omega)=\frac{k\left(1+j \omega / \omega_{5}\right)}{\left(1+j \omega / \omega_{3}\right)\left(1+j \omega / \omega_{4}\right)} \quad \omega_{4}>\omega_{3} .
$$

\section{B. Common-Mode Stability of a Gyrator}

For the stability of the CM positive feedback of a gyrator, the CMFB amplifier needs extra attention. Gyrators are normally loaded with two capacitors to construct a resonator. For 
simplicity let's assume that the value of these two capacitors is the same. So if $H(j \omega)$ is the CM transfer function of the OTA with its CMFB amplifier which is loaded with a capacitor $2 c_{L}$ (see Figure 3), the block diagram of the CM representation of a resonator can be drawn as in Figure 4.

From Figure 4 the $\mathrm{CM}$ transfer function of the resonator $(T(j \omega))$ can be written as:

$$
T(j \omega)=\frac{H(j \omega)}{1-H^{2}(j \omega)}=\frac{H(j \omega)}{[1-H(j \omega)][1+H(j \omega)]} .
$$

It seems from (5) that $T(j \omega)$ consists of the cascade of a negative and a positive feedback systems. Assuming $H(j \omega)$ is stable by choosing appropriate values for $k$ and $\omega_{4}$, the stability of $T(j \omega)$ can be examined by looking at the Bode plot of the loop gain $H(j \omega)$ of each system. Having enough PM for the stability of $T(j \omega)$ for both positive and negative feedback systems of $H(j \omega)$ is cumbersome and especially due to the parasitic poles and zeroes in the circuit, is tricky to control. Therefore, a sufficient condition to ensure the stability of $T(j \omega)$ is to avoid any zero crossing in the magnitude Bode plot of $H(j \omega)$, i.e.:

$$
|H(j \omega)|=\left|\frac{G_{C M}(j \omega)}{1+F(j \omega)}\right|<1 .
$$

Referring to Figure 3 and by substituting (2) and (4) in (1), the transfer function of $H(j \omega)$ can be rewritten as (7), considering that $\omega_{1}=\omega_{3}$. It can be deduced from (7) that $H(j \omega)$ has one zero and three poles. To locate them on the Bode plot it is helpful to look back at the poles of $G_{C M}(j \omega)$ and $F(j \omega)$. For a differential OTA $G(j \omega)$, the internal pole should be located far beyond its gain-bandwidth product (GBW) [11]. Therefore its dominant and internal poles are fairly separated from each other. Although $\omega_{1}$ and $\omega_{2}$ are the CM poles of $G(j \omega)$, they cannot be far away from their differential counterparts, thus it easily can be assumed that $\omega_{2} \gg \omega_{1}$. The position of the poles and zero of $F(j \omega)$ is taken as in (3) (i.e., $\omega_{4}>\omega_{3}$ ). Based on these assumptions, the relative position of the poles and zero on the frequency axis is shown in Figure 5.

Apart from $\omega_{2}, H(j \omega)$ has two other poles $\left(\omega_{6}\right.$ and $\left.\omega_{7}\right)$. From (7) it can be deduced that they are either negative and real or a pair of complex conjugate poles. Each of these two cases will be described separately.

1) Real Poles: If $\omega_{6}$ and $\omega_{7}$ are real, assuming that $\omega_{6} \leq$ $\omega_{7}$, the pole of interest that may cause instability in $T(j \omega)$ is $\omega_{6}$. In other words, $\omega_{6}$ should compensate for the effect of the zero at $\omega_{4}$ to avoid zero crossing in $H(j \omega)$. Figure 5 shows a magnitude Bode plot of $G_{C M}(j \omega)$ and $F(j \omega)$ as well as $H(j \omega)$ for one case where $\omega_{4}<\omega_{6}<\omega_{2}$ and $k>$ $G_{0}$. If $\omega_{6}$ occurs before $\omega_{4}$ then the effect of the zero at $\omega_{4}$ is compensated for and the condition for no zero crossing is ensured. The problem starts when $\omega_{6}$ takes place after $\omega_{4}$ and rises by increasing the value of $\omega_{6}-\omega_{4}$. The worst case is when $\omega_{6}$ occurs at a higher frequency than $\omega_{2}$, in that case, to ensure the stability the required value of $k$ can be derived as:

$$
20 \log _{10}\left(\frac{1+k}{G_{0}}\right)-20 \log _{10}\left(\frac{\omega_{2}}{\omega_{4}}\right)=G M
$$

where $G M$ is the safety margin to avoid zero crossing due to the approximations used with the modeling of $G_{C M}(j \omega)$ and $F(j \omega)$. Assuming $G M=20 \mathrm{~dB}$ in (8), the minimum value of $k$ can be calculated as

$$
k=\frac{10 \omega_{2} G_{0}}{\omega_{4}}-1,
$$

where $\omega_{4}$ comes from the stability condition of $H(j \omega)$. However, it is preferred to place $\omega_{6}$ somewhere between $\omega_{4}$ and $\omega_{2}$ as shown in Figure 5. In this case it is possible to push $\omega_{4}$ to a lower value and save power.

2) Complex Conjugate Poles: If the system has a pair of complex conjugate poles $\left(\omega_{6}=\omega_{7}^{*}\right)$ close to the imaginary axis, the magnitude of the frequency response has a peak, or resonance at frequencies in the proximity of the pole which for (7) happens at (10). The real part of the resonance frequency can be calculated as (11). Increasing it, i.e. placing $\omega_{6,7}$ away from the imaginary axis of the s-plane, decreases the magnitude of the peak at the resonance frequency.

$$
\begin{gathered}
\left|\omega_{6,7}\right|=\sqrt{(1+k) \omega_{3} \omega_{4} .} \\
R e\left(\omega_{6,7}\right)=\frac{1}{2}\left[\omega_{3}+\omega_{4}+\frac{k \omega_{3} \omega_{4}}{\omega_{5}}\right] .
\end{gathered}
$$

From (10) and (11) it is clear that the frequency and the magnitude of the peaking can be controlled by the value of $k$ and $\omega_{4}$ while $\omega_{5}$ sets the magnitude of the peak, independently. The magnitude Bode plot of $H(j \omega)$ along with $G_{C M}(j \omega)$ and $F(j \omega)$ is sketched by inspection on the position of the poles and zeroes in Figure 6 for the case of the existence of a pair of complex conjugate poles in $H(j \omega)$.

To avoid any zero crossing in the magnitude Bode plot of $H(j \omega)$ in Figure 6, due to the existence of a zero at $\omega_{4}$ in $H(j \omega)$, a high value of $k$ is required. Besides, placing the compensation zero $\left(\omega_{5}\right)$ close to or even before $\omega_{4}$ increases (11) and thus helps to decrease the peaking in the magnitude response of $H(j \omega)$ and avoids zero crossing.

\section{Common-Mode Stability: Design METHODOLOGY}

The mathematical analysis and the requirements to ensure the CM stability of a gyrator was presented in section II-B. In this section, we provide a design methodology for the CM stability of a gyrator that transforms the requirements in section II-B to the more practical ones that can easily be verified during the CAD based CMFB amplifier design. These requirements can be developed fairly easily at high and low frequencies, while the requirements on $F(j \omega)$ for the transition frequency are more difficult to determine. Assuming that $H(j \omega)$ is stable (see section II-A), these requirements are as follows:

1) Stability at low frequencies: At low frequencies, if the magnitude of $F(j \omega)$ is much larger than 1 the following approximations can be made:

$$
|F(j \omega)| \gg 1 \Rightarrow\left|\frac{1}{1+F(j \omega)}\right| \approx\left|\frac{1}{F(j \omega)}\right| .
$$




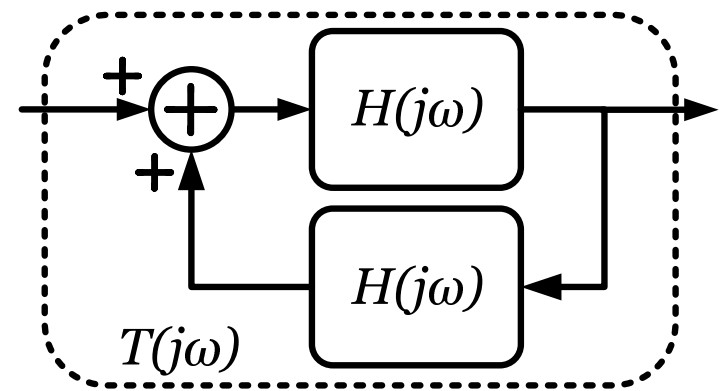

Figure 4. Block diagram of a $\mathrm{CM}$ representation of a resonator; $H(j \omega)$ is the CM transfer function of the OTA with its CMFB amplifier (Figure 3).

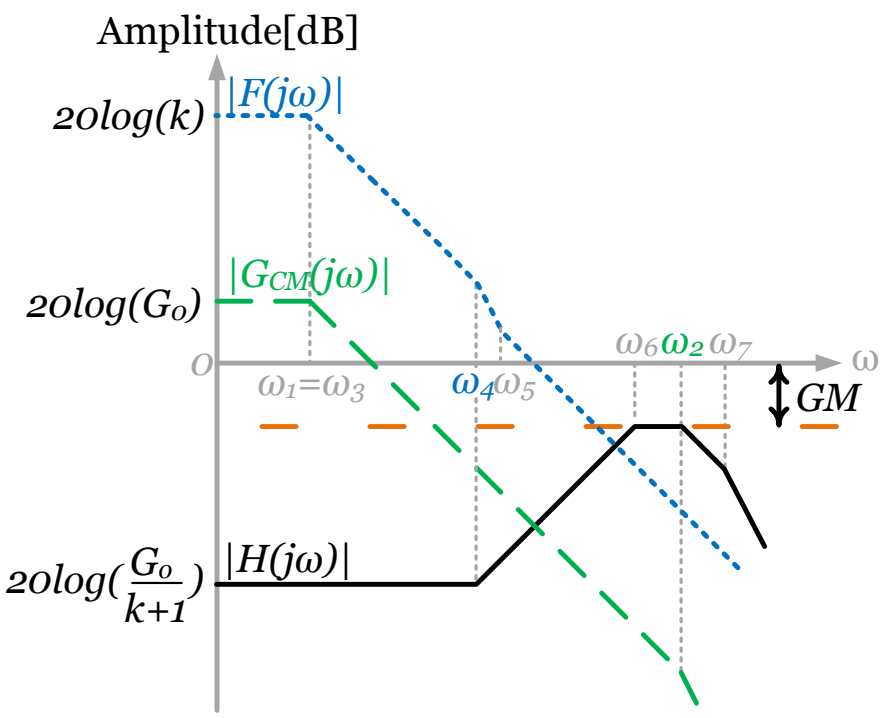

Figure 5. Bode magnitude plot of $G_{C M}(j \omega), F(j \omega)$ and $H(j \omega)$; in case of real poles in $H(j \omega)$

If (12) is used in (6) the following approximate requirement results:

$$
\left|\frac{G_{C M}(j \omega)}{F(j \omega)}\right|<1
$$

Thus, the requirement of (6) is very close to being satisfied at low frequencies if (13) is satisfied. The inequality in (13) means that at low frequencies, a large value of $|F(j \omega)|$ is needed, and that $|F(j \omega)|>\left|G_{C M}(j \omega)\right|$. Hence, a high DC gain for $|F(j \omega)|$ is required. Indeed, this is the equivalent of increasing the value of (8) and (11) to avoid zero crossing in $|H(j \omega)|$.

2) Stability at high frequencies: At high frequencies, if the magnitude of $F(j \omega)$ is much smaller than 1 the following

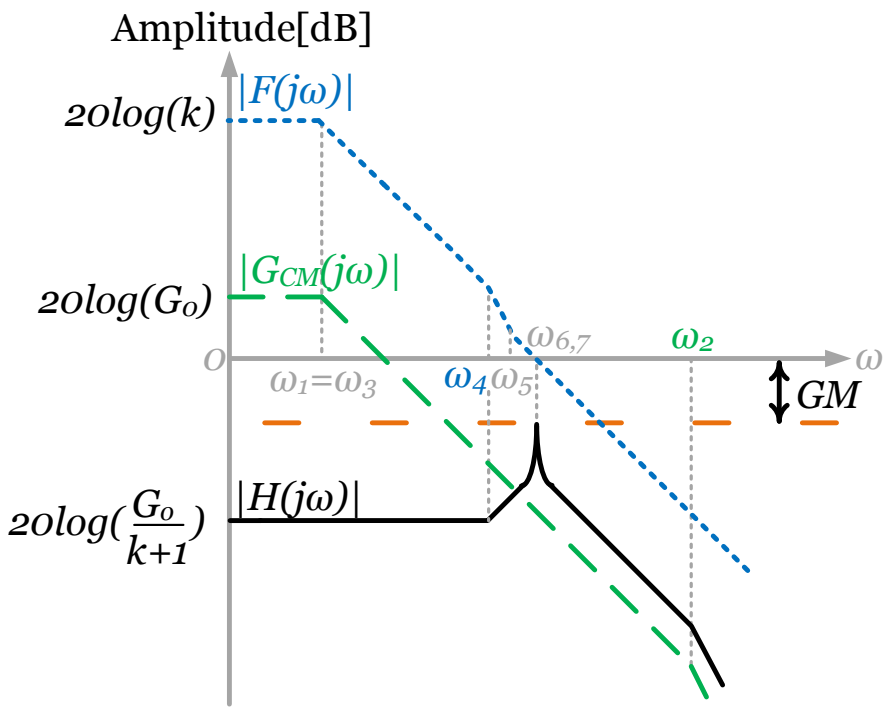

Figure 6. Bode magnitude plot of $G_{C M}(j \omega), F(j \omega)$ and $H(j \omega)$; in case of complex conjugate poles in $H(j \omega)$.

approximations can be made:

$$
|F(j \omega)| \ll 1 \Rightarrow\left|\frac{1}{1+F(j \omega)}\right| \approx 1 .
$$

If (14) is used in (6) the following approximate requirement results:

$$
\left|G_{C M}(j \omega)\right|<1 \text {. }
$$

Therefore, the requirement of (6) is very close to being satisfied at high frequencies if (15) is satisfied. The inequality in (15) means that the CM gain of the OTA should be made small enough at high frequencies, and that $|F(j \omega)| \ll 1$. This condition is commonly satisfied by using resonator's loading capacitors $\left(c_{L}\right)$ connected to GND rather than purely differential load.

3) Stability within the transition band: The requirement of $F(j \omega)$ in the transition band is more difficult to determine because the assumptions leading to (12) and (14) are not valid anymore. However, it is clear that $|F(j \omega)|$ must change from being large at low frequencies to being small at high frequencies, and thus somewhere in the transition band $|F(j \omega)|$ has its crossover frequency $\omega_{c}$ with the $\mathrm{PM} \phi_{m}$ where $\left|F\left(j \omega_{c}\right)\right|=1$. At $\omega_{c}$, we have:

$$
\left|1+F\left(j \omega_{c}\right)\right|=\left|1+e^{j\left(\pi-\phi_{m}\right)}\right|=\left|2 \sin \left(\frac{\phi_{m}}{2}\right)\right|,
$$

By using (16) in (6) the required PM can be derived as

$$
\phi_{m, \min } \geq 2 \arcsin \left(\frac{\left|G_{C M}\left(j \omega_{c}\right)\right|}{2}\right) \text {. }
$$

$$
H(j \omega)=\frac{\frac{G_{0}}{\left(1+j \frac{\omega}{\omega_{1}}\right)\left(1+j \frac{\omega}{\omega_{2}}\right)}}{1+\frac{k\left(1+j \frac{\omega}{\omega_{5}}\right)}{\left(1+j \frac{\omega}{\omega_{3}}\right)\left(1+j \frac{\omega}{\omega_{4}}\right)}}=\frac{\frac{G_{0}}{k+1}\left(1+j \frac{\omega}{\omega_{4}}\right)}{\left(1+j \frac{\omega}{\omega_{2}}\right)\left[1+\frac{j \omega}{(k+1)}\left(\frac{1}{\omega_{3}}+\frac{1}{\omega_{4}}+\frac{k}{\omega_{5}}\right)-\frac{\omega^{2}}{(k+1) \omega_{3} \omega_{4}}\right]}, \begin{aligned}
& \omega_{2}>\omega_{1}, \\
& \omega_{4}>\omega_{3} .
\end{aligned}
$$


Equation (17) determines the minimum required PM, but to ensure the stability practically, it must be made larger than the minimum requirement to bear for process, voltage and temperature (PVT) variations.

\section{Design Procedure For THE COMMON-Mode} STABILITY

Assuming that an OTA has been designed for differential mode performance and is stable, based on the proposed methodology in section III, we present the procedure to design a CMFB amplifier that ensures the CM stability of the gyrator or the resonator constructed by that OTA, as follows:

1) Before starting to design the CMFB amplifier, its topology has to be chosen. Among the different topologies of the CMFB amplifier, one that allows to control the $\mathrm{GBW}_{\mathrm{CM}}$ independently from differential-mode gain-bandwidth product $\left(\mathrm{GBW}_{\mathrm{DM}}\right)$ is preferred. $\mathrm{GBW}_{\mathrm{CM}}$ should be set higher than the $\mathrm{GBW}_{\mathrm{DM}}$ of the main amplifier. Indeed, if the CMFB amplifier were slow, a high-speed spike in the substrate or supply line would put some transistors of the main amplifier in the linear region. The noise or spur generated from digital circuitry or intermodulation (IM) products of an analog part falling within the BW of the main amplifier could cause the same problem. A slow CMFB amplifier would take too much time to restore the biasing, and during that time, the main amplifier would be out of operation. So a CMFB amplifier with a higher GBW than the main amplifier is necessary to avoid this situation [12].

2) Part of the output stage of the main OTA is shared between the OTA and its CMFB amplifier. In fact, CMFB amplifier consists of this shared part and an error amplifier (EA). Therefore, after choosing the topology of the CMFB amplifier, the design narrows down to the design of an EA. A static analysis is necessary to find the required output range of the EA to set the CM voltage for the whole possible range of the CM input. At first an ideal amplifier can be used as an EA to simulate and find this range. It is especially important for the gyrator because the two amplifiers are connected in CM positive feedback. Besides, during the startup of the circuit, the CM voltage will sweep from GND to its nominal value. Therefore, the CMFB amplifier should set the right CM output level for all possible CM input ranges or at least avoid any latch up in the output stage of the main amplifier. After finding this range the EA can be designed to provide this output voltage range. This voltage range is the result of transistors' width ratios in the EA. Indeed, the design in this stage is to set the size of the transistors of the EA to provide this voltage range. The gain and $\mathrm{GBW}_{\mathrm{CM}}$ will be set later. Note that, the ratios of the transistors' width from this stage should be preserved during the rest of the design.

3) Another consideration is the capacitance loading of the CMFB amplifier if connected to GND. The CMFB amplifier sees twice the capacitive load of the main differential amplifier, which directly affects the $\mathrm{GBW}_{\mathrm{CM}}$ and the PM, and should be taken into account during simulation [13].

4) Finally the frequency response of the CMFB amplifier has to be shaped and the $\mathrm{GBW}_{\mathrm{CM}}$ has to be set. The simulation setup to find the $\mathrm{GBW}_{\mathrm{CM}}$ and $\mathrm{PM}$ of the CMFB amplifier needs particular attention due to the fact that open-loop simulations are required. In fact the CMFB loop should be broken for these simulations but opening the loop unbalances the biasing of the circuit. A CMFB amplifier provides biasing for the main amplifier as well as itself since part of the circuit is shared between both of them. Therefore, simulating the CMFB amplifier with a fixed and approximated biasing of the main amplifier is not possible. To that effect, the feedback loop should be closed for the DC signal but of course broken for the AC signal. Opening the loop for a small signal is possible with an ideal loop probe or controlled source along with an ideal low pass filter. Having regard to these considerations as well as stability conditions presented in section III, the design process for the CMFB amplifier continues to shape its frequency response and set its $\mathrm{GBW}_{\mathrm{CM}}$. The frequency response of a CMFB amplifier $(F(j \omega))$ is divided into three regions (low, transition and high frequencies), for which the CMFB amplifier should satisfy some conditions to ensure the CM stability of the gyrator.

At low frequencies, conditions (12) and (13) should be satisfied so a high DC gain for $F(j \omega)$ is required. This can be achieved by increasing the transconductance of the input transistors of the EA which also increase the $\mathrm{GBW}_{\mathrm{CM}}$ of the CMFB amplifier. Note that the transistors' width ratios which were obtained during the static design must be preserved.

At high frequencies, conditions (14) and (15) should be satisfied. The CMFB amplifier has the same loading capacitance as the OTA, except that the OTA sees it in differential mode (i.e. $c_{L}$ ) and the CMFB amplifier sees it in common-mode (i.e. $2 c_{L}$ ). To satisfy (14) and (15) the capacitive load or a part of it should be connected to GND to provide the path also for the CM signals.

At transition frequency, of course the CMFB amplifier should have enough PM for its own stability but to ensure the stability of the gyrator it should satisfy (17) at its unitygain frequency $\left(\omega_{c}\right)$. More PM can be achieved by means of a compensation capacitor which introduces a zero in the transfer function of the CMFB amplifier. In fact, gaining more PM by introducing a zero close to the second pole of $F(j \omega)$ increases (11) and places the $\omega_{6,7}$ away from the imaginary axis of the s-plane.

\section{Case Study: a High Quality Factor, High FREQUENCY RESONATOR}

To clarify and validate the proposed methodology, a resonator with $20 \mathrm{MHz}$ resonance frequency and quality factor around 20 was designed and fabricated using UMC 180-nm complementary metal-oxide-semiconductor (CMOS) technology. The schematic of the test-chip is shown in Figure 7 together with the die photograph in Figure 8. As the differential mode design was presented in [11], here we focus on the design of the CMFB amplifier.

Step 1: The design procedure starts with choosing a value for the loading capacitance because it affects all the parameters for the design of the resonator. Although a lower value of the capacitor works in favor of power consumption and silicon die area, usually its minimum value is set by the noise requirement 


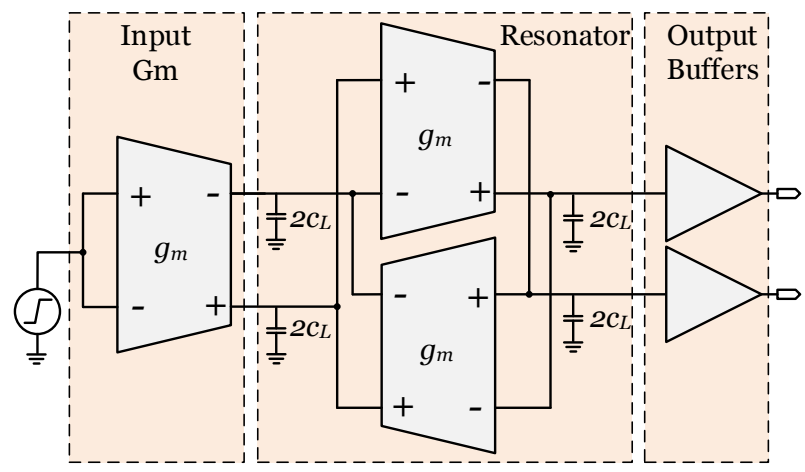

Figure 7. Schematic of the implemented circuit to examine the CM stability of the resonator.

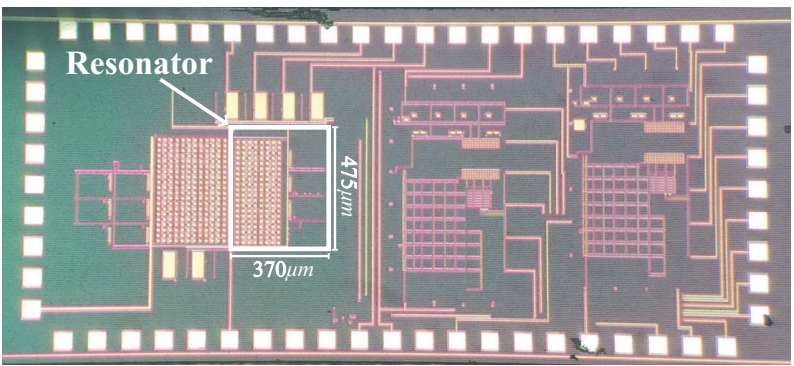

Figure 8. Micro photograph of the chip implemented in UMC 180-nm CMOS technology.

of the resonator [14]. In this design, based on the noise requirement of the resonator, $c_{L}=1.18 \mathrm{pF}$ was chosen. The input and output CM voltages are set to $900 \mathrm{mV}$ (VDD/2) due to the fact that in the resonator the OTAs are connected back to back in a feedback loop. In other words, the output nodes of one OTA is the input nodes of the following OTA thus the output $\mathrm{CM}$ voltage should be fixed to the value of the input $\mathrm{CM}$ voltage.

Step 2: The next step is to choose the topology of the OTA. The OTA's topologies are well explained in [12]. Among the different topologies for OTAs, the folded cascode has three main advantages over the others which makes it the first choice for the most designers. The first advantage is that the $\mathrm{CM}$ input voltage range can reach one of the supply rails. The second advantage is that the output stage always conducts current regardless of the input stage. Therefore, the output $\mathrm{CM}$ can be obtained even if the input $\mathrm{CM}$ would deviate from its typical value. The last advantage which is very important in resonator design, is that the second pole is located at very high frequency compared to other topologies, i.e. telescopic or symmetrical OTA, without any extra compensation techniques. Therefore, the folded cascode topology was preferred for this design. The schematic of the folded cascode OTA is shown in Figure 9. Since the differential mode design is out of scope of this paper here we only report its main parameters. Employing the methodology proposed in [11], the OTA has been designed for differential mode to have $\mathrm{GBW}_{\mathrm{DM}}$ of $20 \mathrm{MHz}$. Its second pole has been placed at $880 \mathrm{MHz}$ resulting in a quality factor of 20. The OTA's core without its biasing circuitry consumes $307 \mu \mathrm{A}$ from $1.8 \mathrm{~V}$. All the sizing and biasing currents are

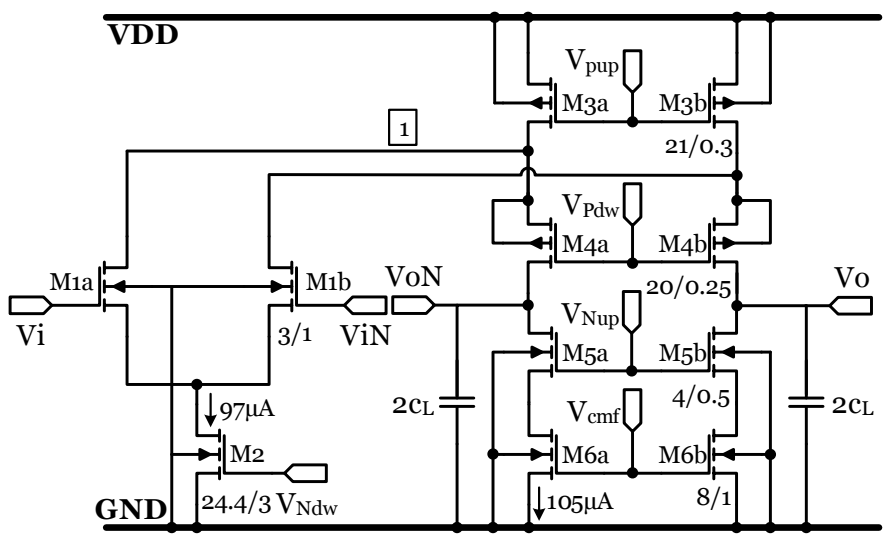

Figure 9. Schematic of the folded cascode OTA with a differential load of $c_{L}$.

shown in Figure 9.

Step 3: After the design of the OTA comes the design of the CMFB amplifier which first design step is to choose its topology. The schematic of the selected CMFB amplifier is shown in Figure 11. It consists of an EA followed by a common-source amplifier that is part of the second stage of the main OTA. The topology in Figure 11 was chosen due to the trade-off between power consumption and the feasibility of setting the $\mathrm{GBW}_{\mathrm{CM}}$ independently from the main OTA. The $\mathrm{GBW}_{\mathrm{CM}}$ depends on the transconductances of MC2a-c and can therefore be set independently from the main amplifier to a higher value than $\mathrm{GBW}_{\mathrm{DM}}$.

Step 4: To find the required output range of the EA to set the CM voltage for the whole possible range of the CM input, an ideal amplifier is used instead of the EA. The simulation result in Figure 10 shows that, for the OTA of Figure 9, the Vcmf (see Figure 9) range of $350-600 \mathrm{mV}$ can set the output $\mathrm{CM}$ at $900 \pm 1 \mathrm{mV}$. For that purpose, without considering the $\mathrm{GBW}_{\mathrm{CM}}$ and the gain, the size of the transistors MC13 have been experimentally determined by changing the width ratio between differential pair and current mirror while all the lengths have been set to $1 \mu \mathrm{m}$. Note that Vcmf applies to the gate of M6, therefore the length of M6 should be large enough to avoid channel length modulation.

Step 5: The differential capacitive load $c_{L}=1.18 \mathrm{pF}$ is implemented with two $2 c_{L}$ capacitors connected to the ground to provide the path also for the CM signal. Although, it takes four times the die area than implementing it as a differential load it reduces the parasitics at each output node of the OTA and makes the design more symmetrical.

Step 6: The rest of the design is to shape the transfer function of the CMFB amplifier in low and high frequencies according to (12)-(15). Also the $\mathrm{GBW}_{\mathrm{CM}}$ should be bigger than $\mathrm{GBW}_{\mathrm{DM}}$ which is $20 \mathrm{MHz}$. The $\mathrm{GBW}_{\mathrm{CM}}$ was chosen as $30 \mathrm{MHz}$. These conditions define the bias current of the CMFB amplifier. Setting this current is possible by changing the number of fingers of MC1-3 while keeping the same width ratio determined in step 4 to preserve the output voltage range of the EA. The DC gain of the CMFB amplifier is set to $82 \mathrm{~dB}$ to satisfy (12) and (13), and connecting the loading 


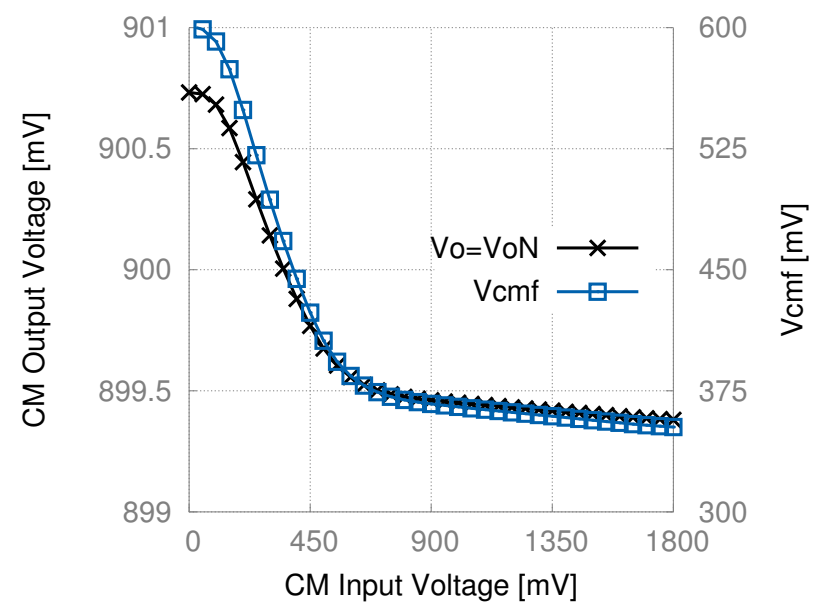

Figure 10. Required range of $\mathrm{V} c m f$ to set the output common-mode.

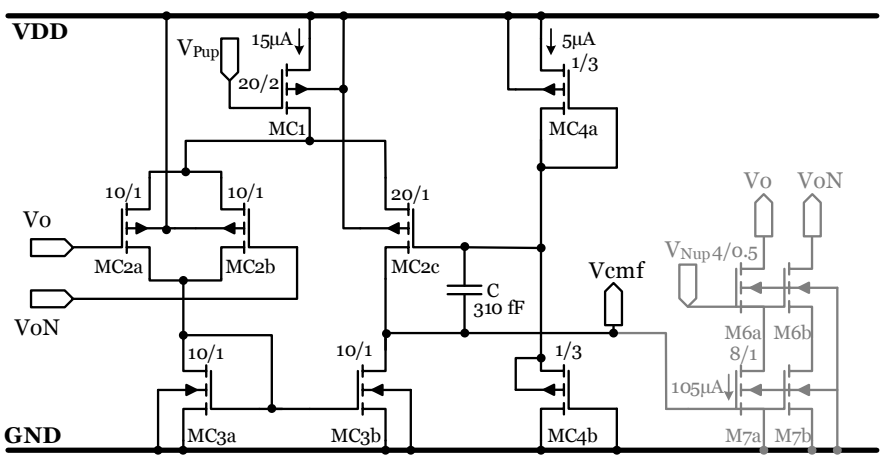

Figure 11. Common-mode feedback amplifier with the error amplifier in black.

capacitance to ground as it is explained in step 5, helps to meet the conditions (14) and (15). Finally, as the last requirement, the PM of $F(j \omega)$ should be larger than (17). Capacitor C along with transistors MC4 in Figure 11 introduce a zero to the transfer function of the EA and the PM of the circuit can be easily adjusted by tuning the size (wd) of MC4 and C. All the sizing and biasing currents are shown in Figure 11. The simulation results of the CM gain and phase of the main OTA as well as the CMFB amplifier are shown in Figure 12.

It is clear that the requirements of $F(j \omega)$ and $H(j \omega)$ for the CM stability of $T(j \omega)$ are satisfied (see section II) at low and high frequencies. At crossover frequency $\left(\mathrm{GBW}_{\mathrm{CM}}\right.$ or $\left.\omega_{c}=30 \mathrm{MHz}\right),\left|G_{C M}\left(j \omega_{c}\right)\right|=-33 \mathrm{~dB}$; thus from (17) the minimum required $\mathrm{PM}$ is around $1.3^{\circ}$ which is much less than the achieved $50^{\circ} \mathrm{PM}$ for $F(j \omega)$. Simulation results in Figure 13 show that reducing the PM by tuning the width of MC4 can cause zero crossing in $H(j \omega)$ which leads to instability of $T(j \omega)$.

The measured response of the implemented resonator is shown in Figure 14. It shows a quality factor of 22 with a resonance frequency at $20 \mathrm{MHz}$. The resonator consumes 660 $\mu \mathrm{A}$ from $1.8 \mathrm{~V}$. To apply the signal to the resonator the same OTA as in the resonator is used at the input (see Figure 7). Two output buffers are employed to avoid loading the resonator

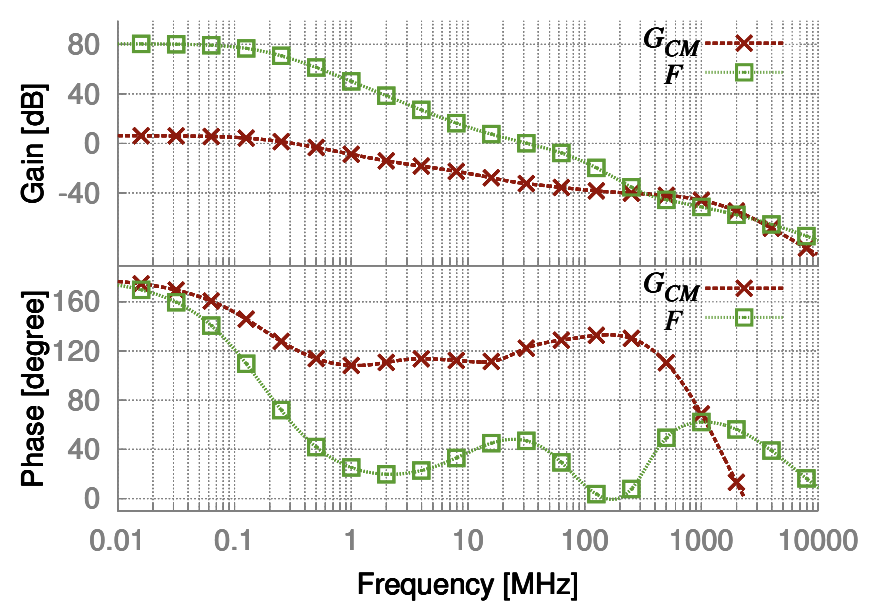

Figure 12. Simulated CM gain and phase of the main OTA $\left(G_{C M}(j \omega)\right)$ and the CMFB amplifier $(F(j \omega))$.

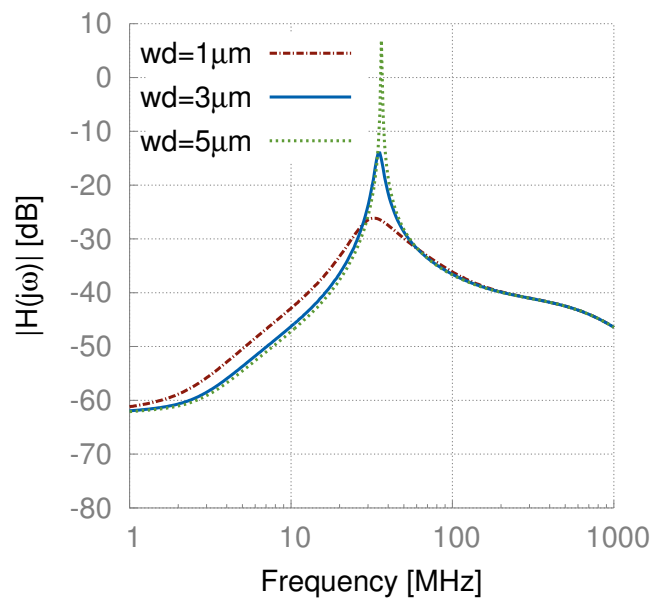

Figure 13. Effect of tuning the width (wd) of MC4 on the CM stability.

with measurement instruments. To examine the CM stability, a step signal with an amplitude of $300 \mathrm{mV}$ was applied to the OTA's input as a CM signal. The resonator's response to the step input is shown in Figure 15. It is clear that the CMFB amplifier acts instantly to set the output $\mathrm{CM}$ voltage (settling time $<20 \mu \mathrm{s}$ ) and the resonator is completely stable.

\section{CONCLUSION}

In this paper, the topology of the OTA-based gyrator, which is commonly used in active resonators, is briefly reviewed. It was shown that a stable OTA is not always sufficient to guarantee the $\mathrm{CM}$ stability of a gyrator. Indeed due to the feedback loop in the architecture of the gyrator, more stringent requirements than just the CM stability of the OTA must be satisfied. To that effect, the mathematical models for $\mathrm{CM}$ analysis of a gyrator were presented and the sufficient requirements for its $\mathrm{CM}$ stability were analyzed. Based on these requirements, the methodology of how to design a CMFB amplifier for a given differential OTA employed in a resonator 


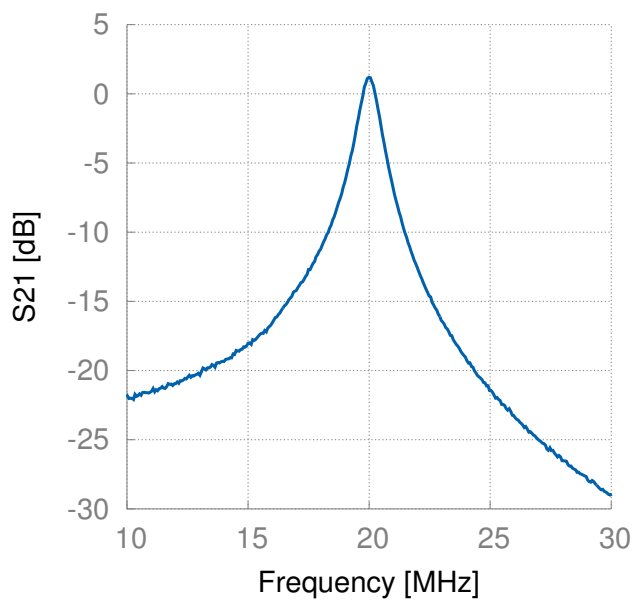

Figure 14. Measured resonator response tuned at $20 \mathrm{MHz}$, estimated quality factor is 22 .

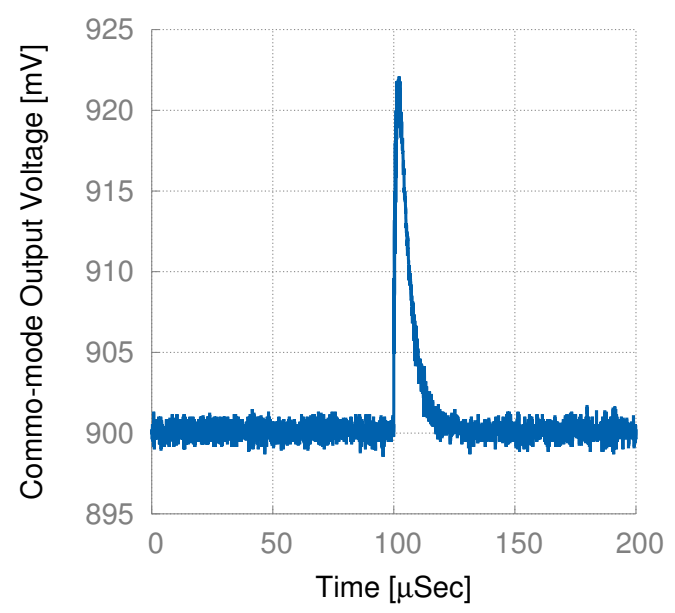

Figure 15. Measured resonator CM response to a $300 \mathrm{mV}$ step signal applied as a $\mathrm{CM}$ signal to the resonator input.

was presented. The methodology ensures the CM stability of the OTA as well as the resonator. The proposed methodology yields to the detailed procedure that facilitates the design of a CMFB amplifier of a gyrator or a resonator. The simulation procedure to find the PM and GBW of the CMFB amplifier was also described. The methodology was employed for design and fabrication of a CMFB amplifier for a resonator. Finally, a measurement result shows the complete stability of the resonator.

\section{ACKNOWLEDGMENT}

The authors would like to thank Prof. Federico Alimenti, University of Perugia, Italy, and Prof. W. M. C. Sansen, Catholic University of Leuven, Belgium, for their valuable comments on this work.

\section{REFERENCES}

[1] Qi, N., Xu, Y., Chi, B., Yu, X., Zhang, X., Xu, N., Chiang, P., Rhee, W., and Wang, Z., A Dual-Channel Compass/GPS/GLONASS/Galileo Reconfigurable GNSS Receiver in $65 \mathrm{~nm}$ CMOS With On-Chip I/Q Calibration, IEEE Transactions on Circuits and Systems I: Regular Papers, vol. 59, no. 8, pp. 1720-1732, Aug. 2012. DOI: 10.1109/TCSI.2012.2206502

[2] Xie, H., Oliaei, O., Rakers, P., Fernandez, R., Xiang, J., Parkes, J., Riches, J., Verellen, R., Rahman, M., Bhan, V., and Schwartz, D. B., Single-Chip Multiband EGPRS and SAW-Less LTE WCDMA CMOS Receiver With Diversity, IEEE Transactions on Microwave Theory and Techniques, vol. 60, no. 5, pp. 1390-1396, May 2012. DOI: 10.1109/TMTT.2012.2187796

[3] Morche, D., Masson, G., De Rivaz, S., Dehmas, F., Paquelet, S., Bisiaux, A., Fourquin, O., Gaubert, J., and Bourdel, S., Double-Quadrature UWB Receiver for Wide-Range Localization Applications With Sub-cm Ranging Precision, IEEE Journal of Solid-State Circuits, vol. 48, no. 10, pp. 2351-2362, Oct. 2013. DOI: 10.1109/JSSC.2013.2272352

[4] Chastellain, F., Botteron, C., and Farine, P.-A., Looking Inside Modern Receivers, IEEE Microwave Magazine, vol. 12, no. 2, pp. 87-98, Apr. 2011. DOI: 10.1109/MMM.2010.940105

[5] Yuan, F., CMOS gyrator-C active transformers, IET Circuits, Devices \& Systems, vol. 1, no. 6, p. 494, 2007. DOI: 10.1049/iet-cds:20070103

[6] Zhang, X. and El-Masry, E. I., A Novel CMOS OTA Based on Body-Driven MOSFETs and its Applications in OTA-C Filters, IEEE Transactions on Circuits and Systems I: Regular Papers, vol. 54, no. 6, pp. 1204-1212, Jun. 2007. DOI: 10.1109/TCSI.2007.897765

[7] Liscidini, A., Pirola, A., and Castello, R., A $1.25 \mathrm{~mW}$ 75dB-SFDR CT filter with in-band noise reduction, in 2009 IEEE International Solid-State Circuits Conference-Digest Technical Paper. IEEE, Feb. 2009, pp. 336-337,337a. DOI: 10.1109/ISSCC.2009.4977445

[8] Handkiewicz, A., Katarzyski, P., Szczsny, S., Wencel, A., and Śniataa, P., Analog filter pair design on the basis of a gyratorcapacitor prototype circuit, International Journal of Circuit Theory and Applications, vol. 40, no. 6, pp. 539-550, 2012. DOI: 10.1002/cta.741

[9] Antoniou, A., Stability properties of some gyrator circuits, Electronic Letter, vol. 4, no. 23, p. 510, 1968. DOI: $10.1049 / \mathrm{el}: 19680396$

[10] Monsurr, P., Pennisi, S., Scotti, G., and Trifiletti, A., High-tuning-range CMOS band-pass IF filter based on a low-Q cascaded biquad optimization technique, International Journal of Circuit Theory and Applications, pp. n/a-n/a, 2014. DOI: 10.1002/cta.2027

[11] Ghamari, S., Tasselli, G., Botteron, C., and Farine, P.-A., Design Methodology for Controlled-Q Resonators in OTA-based Filters, in Proceedings of IEEE Radio Frequency Integrated Circuits Symposium. Phoenix: IEEE, May 2015, pp. 371-374.

[12] Sansen, W. M. C., Analog Design Essentials, ser. The International Series in Engineering and Computer Science. Boston, MA: Springer US, 2006, vol. 859. DOI: 10.1007/b135984

[13] Lo, T.-Y. and Hung, C.-C., A 1 GHz Equiripple Low-Pass Filter With a High-Speed Automatic Tuning Scheme, IEEE Transactions on Very Large Scale Integration (VLSI) System, vol. 19, no. 2, pp. 175-181, Feb. 2011. DOI: 10.1109/TVLSI.2009.2034527

[14] Khorramabadi, H. and Gray, P., High-frequency CMOS continuoustime filters, IEEE Journal of Solid-State Circuits, vol. 19, no. 6, pp. 939-948, Dec. 1984. DOI: 10.1109/JSSC.1984.1052249 